\title{
Trabajo Social en un centro de Servicios Sociales: una familia con 70 gatos
}

\author{
Social Work in a Social Services Center: a family with seventy cats
}

\author{
Lucía SANTOS SANTOS \\ Ayuntamiento de Madrid \\ luciasans@gmail.com \\ Juan Carlos Chans Pousada \\ Ayuntamiento de Madrid \\ jcchans@gmail.com \\ Araceli CANTERO SAIZ \\ Ayuntamiento de Madrid \\ aracansa@gmail.com \\ Iefkine HAMPARZOUMIAN MONTILlA \\ Aldeas Infantiles, Madrid \\ iefkine@gmail.com
}

Recibido: 10/10/2013

Revisado: 09/01/2014

Aceptado: 11/04/2014

Disponible on line: 27/06/2014

La cualidad de sentir y demostrar un interés henchido de concentración en el problema individual de cada cliente es una condición fundamental para la buena práctica del trabajo social de casos.

\section{Resumen}

(Mary E. Richmond, Diagnóstico Social, 1917)

Este artículo comparte una experiencia de intervención familiar con dos personas adultas, madre e hija, inmersas en una situación crítica de aislamiento y vulnerabilidad social. El contexto institucional de la intervención, se sitúa en una Unidad de Trabajo Social de Zona de un Centro de Servicios Sociales de una gran ciudad. La demanda de intervención proviene de una comunidad de vecinos.

Al hilo de la narración del caso, se analizan las posibilidades de un Trabajo Social centrado en las capacidades de la familia, la importancia del encuentro y la relación con las personas expuestas a la situación que se describe. Se enfatiza en la necesidad de promover intervenciones programadas de seguimiento, que reduzcan las actuaciones de urgencia de los profesionales. Todo ello en un momento histórico, marcado por unas condiciones sociopolíticas que enfrentan al sistema público de Servicios Sociales a una amenaza de desmantelamiento hasta ahora inéditos.

Palabras clave: colaboración-exclusión social, Trabajo Social, entrevista empática, vínculo, capacidades familiares.

\begin{abstract}
This article shares an experience of family intervention with two adults, mother and daughter, immersed in a critical situation of social isolation and vulnerability. The institutional context of the intervention is located in a Social Work Unit Area of a Social Service Centre of a large city.

The intervention is implemented under demand of a neighboring community.

In line with the narrative of the case, the chances of a Social Work focused on the capabilities of the family, the importance of meeting and relating to people exposed to the situation described are analyzed.

It emphasizes the need to promote planned monitoring interventions in order to reduce emergency actions professionals. All this in a historical moment marked by socio-political conditions facing the public system of social services to a threat of dismantling hitherto unknown.

Keywords: context of collaboration, social works techniques, social fragility and vulnerability, link value, family capabilities, social services.
\end{abstract}

Referencia normalizada: Santos Santos, L., Chans Pousada, J.C., Cantero Saiz, A., y Hamparzoumian Montilla, I. (2014): «Trabajo Social en un centro de Servicios Sociales: una familia con 70 gatos». Cuadernos de Trabajo Social, 27(1): 71-80.

Sumario: Introducción. 1. Trabajo Social de caso. Una experiencia práctica. 2. Conclusiones. 3. Referencias bibliográficas. 


\section{Introducción}

El presente artículo es el último trabajo del grupo de autores que desarrollan, desde el año 2008, acciones para contribuir a reflexionar y divulgar el Trabajo Social de casos que se realiza a diario en los centros de Servicios Sociales de Atención Social Primaria. La secuencia de estas acciones se inició en un Seminario sobre Trabajo Social de casos, dentro del Programa Formativo del Colegio Oficial de Trabajadores Sociales de Madrid, entre los años 2008 y 2010 (Véase en Breves reseñas biográficas del presente número).

Para todos ellos el objetivo ha sido contribuir a la necesaria sistematización del Trabajo Social de casos a partir de la práctica diaria, para profundizar en el desarrollo de la intervención social directa con personas y familias. Así como alejarse del discurso imperante en algunos contextos profesionales, de pérdida de sentido y funciones propias del Trabajo Social, para aproximarse a un conocimiento más científico de la profesión.

La metodología del grupo de trabajo se articula en el debate y la reflexión, consensuando continuamente conceptos, referencias bibliográficas e ideas. En esta dinámica es difícil permanecer ajenos al contexto político y económico en que se desarrolla actualmente la actividad profesional, dentro de unos Servicios Sociales públicos fuertemente amenazados.

Con el caso que a continuación se desarrolla, los autores quieren, una vez más, dar cuenta de ese Trabajo Social esencial que transciende a todo contexto, a la vez que su estudio lleva a identificar las amenazas que se ciernen sobre la población más vulnerable, cuando las prioridades políticas se alejan de las personas.

\section{Trabajo Social de caso. Una experiencia práctica}

\subsection{Antecedentes y descripción del caso}

Se trata de una familia formada por María (63 años), que convive con su única hija, Manuela, (37 años). Cuando accedieron por primera vez a Servicios Sociales todavía vivía con ellas Antonio (82 años), pareja de María y padre biológico de Manuela, pero a quien nunca reconoció legalmente, aunque conviviera con ellas hasta su fallecimiento.
En la reconstrucción para este artículo de los antecedentes que constan en la historia social del caso, se destacan los siguientes datos:

Existe constancia de una actuación policial con motivo de una situación de violencia verbal de Antonio con su pareja. En el informe policial se describen datos relacionados con un posible cuadro depresivo de María, así como, la negación de Antonio de que existieran problemas en su familia.

Constan diferentes citaciones a la familia, motivadas por denuncias de los vecinos, por las condiciones de insalubridad de la vivienda (malos olores, cucarachas, presencia numerosa de gatos).

No es posible acceder a la familia, no abre la puerta a la policía ni a los Servicios Sociales, ni tampoco a los vecinos, quienes informan que nunca salen a la calle durante el día.

Se trata de un periodo de actuación de 5 años, caracterizado por la intermitencia de la intervención de Servicios Sociales; en la que la familia aparece en momentos de crisis y vuelve a desaparecer en otros periodos. Servicios Sociales pone en conocimiento de la Fiscalía la situación de la familia, por si procediese la adopción de alguna medida protectora.

El fallecimiento de Antonio marca un punto de inflexión en el caso, al reaparecer la familia en Servicios Sociales y permitir, por primera vez, desarrollar una intervención continuada y de una gran intensidad, que se realizará en coordinación con Salud Mental. Va encaminada a abordar la detección de graves indicadores de exclusión social crónica, y a redescubrir y reactivar las capacidades también presentes en madre e hija.

\subsection{Desarrollo de la intervención}

El primer contacto con la familia data de 2008, se realiza en la propia vivienda, con una visita a domicilio a petición de diferentes servicios, y con motivo de la denuncia vecinal de las condiciones de insalubridad anteriormente descritas.

Asistieron: la Policía Municipal y los Servicios de Medio Ambiente, quienes tiraron por la ventana las bolsas de basura, con las que llenaron un camión; así como los operarios de la perrera municipal para recoger a los gatos. El presidente de la comunidad de vecinos informó que la familia no molestaba, pues no era ruido- 
sa, si no fuera por los olores. Al tratarse de un edifico de viviendas sociales, algunos vecinos pidieron al Instituto de la Vivienda el traslado a otro bloque, muy preocupados por su salud ante la gran cantidad de gatos de esta familia.

La vivienda figura a nombre de Antonio, recientemente fallecido, con una superficie de 40 metros cuadrados, consta de un baño en el que no se puede entrar y con el agua cortada por las averías sin reparar que mojaban a los vecinos; una cocina grasienta en la que no cocinan, ya que utilizan un camping-gas, y carecen de nevera. Hay, además, una habitación a la entrada con montones de bolsas y periódicos, y la segunda habitación ciega y sin puerta, que da al cuarto de estar, donde tienen una cama en la que hay tres filas de gatos tumbados encima. Es la misma cama en la que duermen ambas mujeres.

Toda la vivienda ha sido «tomada» por los gatos. En el cuarto de estar, los animales rondan por encima de los muebles y rozan con las patas la cabeza de la profesional. La única ventana está rota y tapada con una tabla para que no se escapen. No tienen agua caliente ni luz, pues los cables están mordisqueados y rotos.

Mientras la protectora de animales se encierra en la vivienda para capturar a los gatos, transcurre la primera entrevista con Manuela y María en el pasillo, a la puerta de la casa, conteniendo la náusea por el hedor a excrementos felinos que invade todas las habitaciones.

La primera pregunta es: ¿cómo habéis llegado a esto? Manuela, responde que se les ha ido de las manos, empezaron con dos gatos y..., (se encoje de hombros) mira desolada a su alrededor.

La siguiente pregunta es: ¿Cómo es qué hoy habéis permitido la entrada a los servicios sanitarios y demás personal, después de tanto tiempo con este problema?

Explican que ya no sabían qué hacer, los gatos seguían reproduciéndose y tenían algunos enfermos a los que tenían que cuidar.

A continuación se les pregunta cómo se sentían en ese momento, María responde que con mucha vergüenza, y Manuela que ella se sentía confundida ante tanta gente desconocida, entre tantos gatos, y con todas las voces que oía.

Se intentó tranquilizarlas, alabándoles su valentía al permitir la entrada de tantas personas en su casa, por dar ese primer paso para mejorar su situación. María seguía hablando de la vergüenza y de la preocupación por si le habrían tirado cosas importantes. Luego hablaron sobre la preocupación que le creaban los gatos y sobre qué iba a ser de ellos. Entonces se les presentó al responsable de las personas que estaban recogiendo a los gatos $\mathrm{y}$, con este gesto, se quedaron más tranquilas.

Se les invitó a ir al Centro de Servicios Sociales al día siguiente, para ver qué necesitaban, para poder conocerse con más calma con la profesional, y ver juntas la forma de que pudieran «utilizarla» mejor. Eso sí, con muchas dudas de que fueran a acudir y también desconociendo hasta qué punto podría tener sentido que ellas fueran al Centro en medio de aquella experiencia.

En este punto encajarían algunas consideraciones de. Rossel (1989), cuando explica que:

Para poder estudiar de una manera creativa las diferentes y complejas situaciones que se presentan al trabajador social (...) es necesario poseer unas herramientas, unas guías mentales sobre las que establecer posibles relaciones entre los elementos que aporta la información del cliente, relaciones que permitan estructurar hipótesis y a partir de las que se irá pidiendo más información. Es necesario también un interés, una motivación para estudiar, para descubrir, para investigar (p. 99).

Con los primeros datos obtenidos en la visita domiciliaria y la observación realizada, se avanza una primera hipótesis que guiará las actuaciones posteriores.

\section{Hipótesis preliminar}

Se trata de una familia que posiblemente se encuentra en unas circunstancias de importante deterioro personal, a la vista de la situación de grave insalubridad ambiental que ha generado, con riesgo para sus miembros y para la comunidad vecinal. Es necesario intervenir de forma inminente y valorar si será necesaria o no la intervención protectora de la Fiscalía.

En España durante mucho tiempo se empleó sin distinción el término «servicio social» o «trabajo social» y quizás en esta doble concepción yace la filosofía de unos servicios sociales que, para muchos, va más allá de un repertorio de recursos. Para otros, no menos numerosos, se quedan ahí (Colom, 2008, p. 51). 
Esta reflexión de Colom permite empezar a desgranar esta experiencia práctica en el contexto de unos Servicios Sociales públicos, tan alabados como denostados y criticados.

Ante esta situación de urgencia emergente, la trabajadora social inicia una actuación cuyo eje principal de intervención radica en la importancia de la acogida y del vínculo con la familia desde el primer momento. Es cierto que la demanda no surge de la iniciativa de la usuaria, sino que se canaliza a través de agentes de redes sociales, que son quienes ponen sobre aviso a la institución.

El primer encuentro se mantiene en el propio entorno familiar, («en el pasillo, a la puerta de la casa») en un momento de gran angustia para todos, familia y profesionales. Hay que enfatizar la afirmación «para todos», ya que en ese momento inicial de la intervención se despliega una relación de incertidumbre y de temor compartido por ambas partes: temor de los profesionales a lo que se puedan encontrar; temor a aquello que las familias pudieran llegar a exigir en determinadas situaciones; y temor al hecho de saber dar una respuesta adecuada ante la repercusión social y mediática que alcanzan este tipo de casos.

No muy lejos de esta sensación, al otro lado, la familia también siente temor a no saber qué va a pasar, si se les va a expulsar de su entorno, o si se les va a comprender y apoyar.

Después de este encuentro «emocional», la trabajadora social estructura la entrevista con ambas mujeres, encuadrando dos grandes principios del Trabajo Social: el respeto y la aceptación de la persona y la ausencia de juicios de valor. Prevalece una relación de cercanía y empatía, en la que se define una intervención adecuada a las particularidades de esta familia, en este momento. El manejo de estas técnicas propias del Trabajo Social define el siguiente momento de la intervención.

\subsubsection{Primera fase de la intervención}

Entrevista en el Centro de Servicios Sociales. La demanda que ellas No traían

Era un lunes y se facilitó una cita a última hora de la mañana $y$, contra todo pronóstico, llegaron. A esta entrevista acudieron madre e hija e informaron que habían aparecido otros 7 gatos más. Explicaron que no habían pedido ayuda antes, por la vergüenza que sentían.

En el transcurso de la entrevista contaron que mantenían una relación con una voluntaria desde hacía 7 años, a quien veían cada quince días, ya que organizaba reuniones con un grupo de personas. Dieron su permiso para hablar con ella y para llamarla en ese momento de la entrevista. La voluntaria, que era trabajadora social, desempeñaba actividades en una parroquia dedicada a atender a personas sin recursos y en situación de exclusión social. Contó que las conocía desde hacía mucho tiempo. Su apoyo había consistido en proporcionarles ropa y un acompañamiento para realizar gestiones administrativas. En todo este proceso se había logrado la tramitación y concesión de la pensión no contributiva para Manuela. Todo ello, siguiendo el propio funcionamiento familiar de intermitencia en los contactos y en los compromisos.

María y Manuela contaron que además recogían comida caducada en los supermercados, pues el mantenimiento de los gatos era muy caro y también comían ellas aquellos productos en buen estado.

El hedor de las dos mujeres era insoportable, pero su elocuencia en aquel momento animó a la profesional a seguir preguntando. Manuela no acudía a Salud Mental desde hacía muchos años, al parecer en algún periodo asistieron de forma algo más regular. Se apreciaba en la relación entre ambas un fuerte apego con escasa diferenciación personal y se observaba un continuo cuidado mutuo.

Se abordó, por lo tanto, el estado de salud de ambas: con Manuela, se habló de sus «voces»y sus «demonios». Relataban episodios en los que a veces a María le daban desvanecimientos, momentos en los que Manuela se asustaba mucho. De vez en cuando acudían al Centro de salud de Atención Primaria por medicación.

La dinámica generada en esta primera entrevista entronca con el señalamiento que aporta Rossel (1989):

El diagnóstico sobre qué tipo de ayuda puede admitir el cliente, en qué momento y de qué manera lo puede aceptar, es tan necesario como el que él [en este caso madre e hija] hace sobre la misma situación y sobre la necesidad de ayuda (p. 143). 
Al finalizar la entrevista, se les pidió permiso para hablar con los profesionales del Centro de salud tomaron acuerdos como: seguir hablando para la semana siguiente, y que lo primordial era empezar por desinfectar la vivienda.

La profesional se va haciendo cargo, a través de este acuerdo de desinfección de la vivienda, de dar respuesta a una demanda que, tal vez, ellas no traían, pero que era manifiesta, a través de la acumulación de suciedad y gatos en la vivienda - síntomas qué duda cabeque apuntan a la existencia un problema psíquico mucho más complejo o, tal vez, una disfunción en la dinámica familiar entre madre e hija, que no se puede perder de vista en el diagnóstico. Se identifica una presentación del síntoma: el mediáticamente denominado «síndrome de Diógenes», y se interpreta como una forma de expresión de la demanda hacia el otro, en una aproximación psicoanalítica del término.

Sin duda, todo diagnóstico lleva aparejado un plan de actuación, que plan de acción de urgencia, tras la primera entrevista sobre las necesidades más prioritarias consensuadas con las interesadas, cuyo desarrollo fue el siguiente:

- Desinfección de la vivienda.

- Ayuda económica para pasar una noche mientras limpian la vivienda.

- Coordinación con salud para conducirlas a un reconocimiento médico.

- Seguir construyendo la relación con ellas. Importancia de asegurar la vinculación.

\section{Actuaciones entre entrevistas}

- Supervisión con la Directora del Centro de Servicios Sociales para valorar la ayuda económica.

- Coordinación con el Departamento competente para programar la desinfección en función del resto de acciones.

- Informe social, solicitando la desinfección de la vivienda.

- Limpieza de choque, mediante el Servicio de Ayuda a Domicilio.

- Coordinación con la trabajadora social del Centro de Salud.

- Coordinación de estrategias para conseguir que reanuden el tratamiento con trabajadora social de Salud Mental. Programación de visita a domicilio conjunta más adelante.
Entrevista: Retazos de una historia familiar Acuden madre e hija para informarles de las gestiones que se están realizando, además el objetivo de esta entrevista es conocer la historia familiar. Cuentan que María pasó parte de su infancia interna en un colegio, que tiene una hermana en la ciudad con la que mantiene escasa relación, a veces acuden a su casa para asearse, en algún momento previo a las consultas médicas. De su historia de pareja informan, que Antonio nunca quiso reconocer a la hija y lo interpretan como una posible reacción de rechazo ante su enfermedad y discapacidad. Consiguieron que Manuela asistiera a un colegio de educación especial, pero recuerdan que fue una experiencia negativa para la niña.

Antonio era mucho mayor que María, 20 años de diferencia, y se trataba de una persona muy machista y autoritaria. La vivienda de protección social estaba solamente a su nombre.

De sus ingresos actuales informan que además de la pensión no contributiva de invalidez de Manuela, obtienen dinero de la venta en la calle de las cosas que recogen en la basura.

Posteriormente hubo otra entrevista con ambas mujeres, cuyo objetivo era realizar con ellas el diseño de intervención social

\section{Formulación de hipótesis diagnósticas preli- minares}

a) Valoración de problemas

- Aislamiento y ausencia de red familiar.

- Deficiencia psíquica y enfermedad mental.

- Problemas de salud ni diagnosticados ni tratados.

- Ausencia de organización doméstica (higiene, alimentación, horarios, etc.).

- Problemas de salubridad graves.

- Escasos ingresos económicos y deudas

b) Puntos fuertes

- Colaboración con los profesionales.

- Disponibilidad de vivienda.

- Cuidado mutuo y afecto entre madre e hija.

- Narraciones de hechos significativos en sus vidas donde se han mostrado competentes.

c) Valoración diagnóstica

Se trata de una familia con problemas importantes de salud sin tratar actualmente, que acu- 
mula objetos y animales en la vivienda, provocando problemas de salubridad y deterioro de sus condiciones de vida, a la vez que graves problemas de convivencia vecinal. Con escasa red social y sin familia extensa, arrastran una historia vital en progresivo aislamiento, llegando a una relación de apego patológico entre ambas. Sus dificultades personales afectan a la capacidad de efectuar una demanda de ayuda, posiblemente por el deterioro personal derivado de los distintos problemas de salud vividos en un ambiente familiar hostil. Ingresos estables pero muy escasos que contribuyen junto al resto de los indicadores descritos a una situación de grave riesgo de exclusión social.

\section{d) Objetivos específicos}

- Mejorar las condiciones de salubridad.

- Aumentar de los ingresos económicos.

- Favorecer la creación de una red social y aumentar la relación de confianza con los recursos y con los profesionales.

- Tratamiento en Centro de Salud Mental.

e) Intervención profesional

- Entrevistas en el despacho.

- Visitas domiciliarias.

- Coordinaciones con Salud.

Acuden puntuales a la cita, y a lo largo de esta entrevista se va reforzando la relación profesional. Repasando los recursos que tienen que retomar, se les recuerda que en algún momento de la historia de la familia han logrado llevar una vida normalizada, y por ello se les fomenta el que saben hacerlo, aunque necesiten acompañamiento, $\mathrm{y}$ valorando positivamente que han dado el primer paso aceptando la ayuda.

Al profundizar en la historia de su vida, María revela que había trabajado de vendedora autónoma, cuando contrajo una deuda con la Seguridad Social, que paga mediante un descuento mensual en su pensión mínima. $\mathrm{Ca}$ be preguntarse quién, con el perfil de esta mujer, cumple de forma tan rigurosa con el pago de una deuda o un tributo actualmente.

También hablan de la imposibilidad de solucionar los problemas con la compañía telefónica, debido al rechazo que provocan con su presencia en la central.

El clima de la entrevista da pie para proponerles una nueva visita en su domicilio, con la trabajadora social de Salud mental. Además se le sugiere a María que conozca al grupo que funciona en el Centro, en de un programa de renta mínima en el que se realizan actividades de ocio, habilidades para mejorar las relaciones y hábitos saludables.

\subsubsection{Segunda fase de la intervención}

Visita a domicilio y sucesivas entrevistas. Trabajo social integrado

Es interesante informar ahora de la existencia del Protocolo de Coordinación Socio-Sanitaria, acordado entre instituciones de Atención Sanitaria (Comunidad Autónoma) y Servicios Sociales (Ayuntamiento), dado que, para este caso, sería una herramienta fundamental de intervención que ayudaría a delimitar funciones y acuerdos profesionales.

La profesional decidió no utilizar el protocolo en sentido estricto, que activaría mediante una fría derivación para valoración psiquiátrica, sin duda necesaria en este caso, pero con el riesgo de perderlas en el camino. En una llamada telefónica a la trabajadora social de $\mathrm{Sa}$ lud mental, se agilizó un encuentro para hablar sobre estrategias válidas con esta familia.

Acuerdan utilizar la visita a domicilio, para realizar este nuevo contacto, en el que era importante que acudiesen ambas, dado que con la profesional de Servicios Sociales habían iniciado una vinculación relacional, que había conseguido al transmitirles su respeto a estas dos mujeres, ante su decisión de vivir a su manera, incluso compartiendo con ellas que, en general a la mayoría de las personas le costaba entender el porqué y el cómo habían decidido vivir como lo hacían.

Rodríguez (2007), a propósito de los efectos que puede causar el caso, en la profesional que le atiende indica lo siguiente:

La intervención reflexiva, o también podríamos decir, la actitud reflexiva implica como decíamos, un proceso por el cual podemos vernos y ver a los demás con ojos diferentes. Compartir sentimientos de marginalidad, evitar identidad unidimensional, ya se trate de poner el énfasis en las diferencias culturales o identitarias o no. Mayor reflexividad implica apertura a las diferencias (p. 128).

Se retrasaron unas semanas en la realización de la visita a domicilio acordada, pues era fun- 
damental esperar para conseguir el objetivo de que acudieran ellas previamente al Servicio de Salud Mental. Era necesaria una intervención que favoreciera la concesión de la autorización de la familia a que pudieran acceder a su domicilio las dos profesionales, una vez familiarizadas con la compañera.

Richmond (1995) proporciona algunos argumentos que prevalecen vigentes para aconsejar los primeros contactos en el domicilio:

El desafío que supone para el trabajador social de casos establecer una relación humana desde el principio (...) en el despacho, los clientes adoptan una actitud defensiva, justificando su visita con respuestas. En el domicilio es el trabajador social el que permanece a la defensiva, mientras que sus anfitriones se sienten en su ambiente (p.187).

El objetivo se consiguió, y el trabajo inicial en paralelo entre los dos servicios fue realmente interesante: mientras en Salud Mental abordaron el apego patológico entre ambas, y consiguieron que Manuela acudiera al Centro de Día psicosocial; de forma complementaria, y a lo largo de sucesivas entrevistas en Servicios Sociales, abordaron temas relacionados con sus sensaciones, por ejemplo: en el metro, en relación a los demás, escucharon como una simple mirada de la gente hacía que Manuela se descontrolara y cómo María la conseguía tranquilizar. Ella empezó a expresar su queja por el continuo cuidado de su hija y como a ella eso le agobiaba. También habló, de su problema con la compañía telefónica y de cómo su aspecto suponía una barrera social.

¿Era esto hacer Trabajo Social? ¿Era hablar de la exclusión social con las protagonistas de esta historia?

\subsubsection{Fase de seguimiento y evaluación}

\section{El tiempo de la familia versus el tiempo de la institución}

La trabajadora social quería entender para poder ayudar y así se lo hizo sentir. Algo que tuvo presente para generar confianza fue que no existieran juicios de valor en ningún momento, les podía ayudar en lo que madre e hija quisieran y decidieran, pues eran ellas las protagonistas de su vida.

Con la perspectiva de hoy, se identifica que fue fundamental ir siempre por detrás de ellas, seguir su proceso con los tiempos que ellas necesitaban. A veces es un sobreesfuerzo en el ritmo que la institución impone, y un desafío a la inmediatez que se espera de la intervención profesional. El tiempo como parte del proceso es un postulado básico que, sin embargo, no siempre se tiene en cuenta en el contexto institucional y tampoco lo tienen en cuenta por los propios profesionales.

Sin embargo, ellas van dejando claro que son las que deciden cuándo, cómo y con quién. Ausloos (2005) muestra la importancia de dejar hacer al tiempo.

También la familia hará sus propias hipótesis, ensayarlas, evaluar los resultados, cambiar las actitudes, sacar conclusiones y volver a empezar de otra manera. Es de esta forma que la familia se implica en un proceso de cambio. Y es esto lo que toma su tiempo (p. 27).

Después de realizar la visita a domicilio y empezar el tratamiento en Salud Mental con Manuela, María acude a una entrevista por primera vez sin su hija, quien ya asiste al Centro de Día psicosocial de Salud mental. Retomando con ella el protagonismo de su vida, se investiga qué quiere hacer y de qué manera ayudarla, no solo como madre. Constatando la inexistente red social, lo que hace que sea mucho más vulnerable.

Se solicita la renta mínima, dado que tendría derecho a una pequeña cuantía diferencial, y así posibilitaría continuar trabajando con ellas evitando que huyeran de nuevo. Fueron necesarias varias entrevistas más para hablar del grupo de renta mínima, para que aceptara conocerlos. Constatando una vez más, que las familias necesitan su tiempo como parte del proceso.

En coordinación con la persona que llevaba el grupo, se aseguró la acogida, para que estuvieran al tanto de algunas de sus circunstancias personales. El contenido del proyecto era perfecto para ella. Orientado a trabajar hábitos básicos en grupo e individualmente para personas en situación de exclusión social. María lo aprovechó inmediatamente. Acompañada por la trabajadora social el primer día, la dejó jugando al parchís. Quedarían para que en entrevistas posteriores contara su experiencia.

María fue aceptada por el grupo y recibió ayuda de ellos en la mejora de la vivienda, 
pues se trabajaba también como grupo de apoyo mutuo. Fue significativo que tras finalizar el Proyecto de renta mínima hubo una interrupción muy prolongada que hizo temer por su continuidad, y se produjera un retroceso en el mantenimiento de las condiciones higiénicas de la vivienda

La elaboración escrita del caso llega hasta la fecha en que la profesional de referencia ha de retirarse del seguimiento familiar, debido a un cambio de funciones, momento que coincide con el cierre de la intervención aquí descrita. El relevo de profesional no ha interrumpido el seguimiento con la familia en el dispositivo hasta hoy, y esta perspectiva permite valorar los importantes cambios alcanzados. Así, en estos tres años los logros han ido de la mano de las necesidades, deseos y capacidades de madre e hija. En el largo historial de relación y trabajo, de todo lo conocido de ellas y todo lo que se han atrevido a experimentar, con el protagonismo que la profesional ha sido capaz de darles en este proceso, se encuentran coincidencias con «el enfoque de la metodología del cambio», que propone Gómez (2009), proyectando

Las dificultades que genera dilucidar entre la frontera del cambio, favorecida por la puesta en marcha de la relación de ayuda, con la frontera de la cronicidad establecida por el usuario (...) donde decide los tiempos de acompañamiento y el cese del mismo (p.93).

Para finalizar, queda el interrogante de estar ante una familia que requerirá siempre un mayor o menor acompañamiento de Servicios Sociales, supeditado, cómo no, a la propia decisión de las personas implicadas.

\section{Conclusiones}

A continuación se exponen algunas cuestiones teórico-prácticas surgidas al grupo redactor en el trabajo desarrollado con este caso.

\subsection{Intervención de urgencia}

Este artículo está basado en la experiencia directa de intervención con la familia de una de las autoras que suscribe el presente trabajo; el caso se propone porque ejemplifica de algún modo situaciones que, con mucha más frecuencia de lo que pudiera parecer, obligan a las trabajadoras sociales de Atención Social Primaria a desplegar una atención de urgencia intensiva. Son familias o personas largamente conocidas en el dispositivo, cuyas características de aislamiento y vulnerabilidad coinciden con el caso descrito. En estas situaciones suele ocurrir que la urgencia acapara toda la atención del profesional en ese momento, suspendiendo temporalmente el resto de seguimientos. Fluyen entonces los sentimientos contradictorios al trabajar arrastrados por la «emergencia», mermándose la capacidad de reflexión y de previsión ante los casos. Además de sentir emociones desencadenantes de una angustia personal ante aquello que los sentidos están percibiendo y que inducen al activismo, quizá desmesuradamente. Sin duda merecerían ser identificados y estudiados detenidamente.

Técnicamente, los resultados a corto plazo son altamente eficaces, movilizándose un sinfín de recursos, quizá por aquello de no dejar nada sin intentar que esté al alcance profesional. Ahora bien, cabe preguntarse si son efectivos los despliegues de gestiones que a veces se promueven, si hay consciencia entre las profesionales, de la frecuencia con la que intervienen de «urgencia», cuando el dispositivo realmente no está definido como tal en su estructura.

El reto pasaría por explorar qué pueden hacer tanto los técnicos de atención directa, como los responsables organizativos de los servicios, para prevenir este tipo de situaciones, programando actuaciones de seguimiento que se adelanten en lo posible, a las crisis que desencadenan en muchas ocasiones intervenciones urgentes, como se constata en el proceso estudiado.

\subsection{Aprovechar las crisis de las familias pa- ra generar una relación de ayuda}

Bajo este epígrafe se sintetizan algunos elementos clave de la intervención social del trabajador social, imprescindibles para enfocarla hacia un «cambio», generando contextos de relación que permitan nuevas posibilidades a las personas inmersas en situaciones de importante exclusión o aislamiento. El grupo promotor de este trabajo ha insistido en publicaciones anteriores, en la necesidad de situar a la persona en el centro de nuestro quehacer profesional compartiendo el planteamiento de intervención de Gastañaga (2007). Sus premisas permiten sostener en el plano teórico el trabajo 
desplegado en la práctica con el caso analizado; el autor refiere

Que revertir procesos de exclusión, de estigmatización, abordar contextos con múltiples problemas o desafíos, es trabajar con experiencias de vida y narraciones propias. Cambiar, transformar los contextos para generar procesos de ayuda en el que emerjan nuevas oportunidades para las personas, implica cierta construcción del otro por parte del profesional, así como una aproximación teórica que haga énfasis en el vínculo, las conexiones y los recursos y fuerzas ocultas de la persona y su contexto (p. 191).

\subsection{Trabajo grupal}

El trabajo con María en el grupo fue fundamental, se sintió aceptada y favoreció el empuje que necesitaba para relacionarse con iguales, y separarse de una manera saludable de la relación con su hija, que había acaparado su atención en los últimos años. Ella aceptó, incluso, que alguno de los asistentes la ayudara en los arreglos básicos de la vivienda. Gesto interpretable como un gran avance en la trayectoria de ésta mujer.

Este grupo se reunía con una frecuencia quincenal, se abordaban temas que sus miembros elegían y consideraban de utilidad: desde habilidades básicas para la convivencia, organización económica y doméstica, consejos de salud, recetas de cocina para una buena alimentación ajustadas a sus bajos ingresos, hasta visitas a recursos y lugares históricos de Madrid. Algunos de ellos apenas habían salido en años del entorno del barrio.

La profesional que llevaba el grupo se coordinaba con la trabajadora social de zona, aportando un punto de vista e imagen de las personas diferente y novedosa. Una faceta que las personas ofrecen funcionando en el grupo, es otra parte de sí mismas alejada de los problemas y las demandas que habitualmente monopolizan la relación profesional en el contexto del despacho.

\subsection{Intervenciones basadas en las capaci- dades de las familias}

Ausloos (2005) hace referencia a que, para referirse a las familias, se recurre siempre a los problemas que presentan, sus disfunciones y no a lo que son capaces de hacer. Señala que «hablar de familia competente es una manera de devolverles su competencia en lugar de considerar sus defectos» (p. 33). El autor añade que esto

Modifica la posición del profesional y por tanto si éste trabaja con una familia capaz de resolver los problemas que se representan, ya no se trata de ocuparse de ella, de cargarse en las espaldas el peso de los problemas de la familia, sino al contrario de activar un proceso en el que la familia podrá observar, experimentar y cambiar (idem).

En una familia tan devastada como la que citamos, no es fácil vislumbrar su competencia, así, cuando la profesional se plantea que en algún momento esta familia fue «normal», está eludiendo el diagnóstico recurrente de la cronicidad para rescatar las capacidades con las que iniciar un mínimo proceso de cambio.

\subsection{El sistema público de Servicios Sociales amenazado}

El caso expuesto muestra una atención social centrada en la persona, donde aparecen procesos de trabajo que superan la mera coordinación y se aproximan a un apoyo social integrado, entre administraciones y agentes sociales. Revelan que los recortes presupuestarios arriesgan la protección y el acompañamiento social a los más excluidos. La calidad de vida de las personas, como esta familia, se ve amenazada por la suspensión temporal de un proyecto que dio sentido y sostén a María mientras asistió al grupo. Y ¿qué decir de la deslocalización de servicios que propugna la nueva la Ley de Reforma Local? ¿Cómo se desarrollará un proceso de acompañamiento social como el expuesto, si no es desde la proximidad garantizada por los Servicios de Atención Social Primaria?

Finalmente queda la pregunta de si quedará reconocido el plus de calidad que aportan las trabajadoras sociales, con su experiencia, y buenas prácticas con el que se vienen compensando cada vez más los déficits institucionales.

Los gatos observan a las criaturas, sus gestos, sus incomprensibles operaciones, durante largas horas. Cómo se hace una cama, se barre el suelo, se hace o deshace una maleta, se cose, se hace media (...) cualquier cosa, ellos miran.

(Doris Lessing, Gatos muy distinguidos,1967). 


\section{Referencias bibliográficas}

Ausloos, Guy (2005). Las capacidades de la familia. Barcelona: Editorial Herder.

Colom, Dolores (2008). El Trabajo Social Sanitario. Atención primaria y atención especializada. Teoría y práctica. Madrid: Editorial Siglo XXI.

Gómez, Rosa. (2009). Los límites en la relación de ayuda: El derecho a decidir cómo vivir. Revista de Trabajo Social Hoy, 57.

Gastañaga, José Luis (2007). Construyendo posibilidades: intervención psicosocial en la escuela: Cuadernos de Trabajo Social, 20, 189-207.

Lessing, Doris (1967). Gatos muy distinguidos. Barcelona: Seix Barral.

Richmond, Mary E. (1995). El caso social individual. El diagnóstico social. Madrid: Editorial Talasa.

Rodríguez, Alfonsa (2007). Más allá de la perspectiva crítica. Cuadernos de Trabajo Social, 20, 117-137.

Rossel, Teresa (1989). La entrevista en Trabajo Social. Madrid. Editorial Hogar del Libro S.A.

Santos Santos, Lucía, Cantero Saiz, Araceli, Hamparzoumian Montilla, Iefkiné y Chans Pousada, Juan Carlos (2011). Trabajo Social de casos en los Servicios Sociales de Atención Primaria, a la luz de las enseñanzas de M. Richmond. Revista de Trabajo Social Hoy, 64, 29-52. 